\title{
LEGENDRE TRANSFORMS AND APÉRY'S SEQUENCES
}

\author{
ASMUS L. SCHMIDT
}

(Received August 27, 1992)

Communicated by J. H. Loxton

\section{Abstract}

This article studies particular sequences satisfying polynomial recurrences, among those Apéry's sequence

$$
a_{n}=\sum_{k=0}^{n}\left(\begin{array}{l}
n \\
k
\end{array}\right)^{2}\left(\begin{array}{c}
n+k \\
k
\end{array}\right)^{2}
$$

which is shown to be the Legendre transform of the sequence

$$
c_{k}=\sum_{j=0}^{k}\left(\begin{array}{l}
k \\
j
\end{array}\right)^{3}
$$

This results in the construction of simultaneous approximations of $\pi^{2} / 8$ and $\zeta(3)$.

1991 Mathematics subject classification (Amer. Math. Soc.): 11B37, 11J13, 33C45.

\section{Introduction}

For a sequence $\left(c_{k}\right)$ we shall consider its Legendre transform $\left(a_{n}\right)$ defined by

$$
a_{n}=\sum_{k=0}^{n} c_{k}\left(\begin{array}{l}
n \\
k
\end{array}\right)\left(\begin{array}{c}
n+k \\
k
\end{array}\right) \text {. }
$$

It should be noticed that each sequence $\left(a_{n}\right)$ is the Legendre transform of a unique sequence $\left(c_{k}\right)$ (cf. Section 2). We shall also consider the sequence of Legendre polynomials belonging to $\left(c_{k}\right)$ defined by

$$
a_{n}(x)=\sum_{k=0}^{n} c_{k}\left(\begin{array}{l}
n \\
k
\end{array}\right)\left(\begin{array}{c}
n+k \\
k
\end{array}\right) x^{k}
$$

(C) 1995 Australian Mathematical Society 0263-6115/95 $\$$ A2.00+0.00 
The classical Legendre polynomials orthogonal on $[-1,0]$ belongs in this way to the sequence $\left(c_{k}\right)$ with $c_{k}=1$.

This article is motivated by the following conjecture (see [10]): For integral $r$, $r \geq 2$, numerical evidence indicates that each of the sequences

$$
a_{n}^{(r)}=\sum_{k=0}^{n}\left(\begin{array}{l}
n \\
k
\end{array}\right)^{r}\left(\begin{array}{c}
n+k \\
k
\end{array}\right)^{r}
$$

is the Legendre transform of an integral sequence $\left(c_{k}^{(r)}\right)$. Challenged by this problem it was noticed by W. Deuber, W. Thumser and B. Voigt (University of Bielefeld) that the corresponding sequence $\left(c_{k}\right)$ for $r=2$ seemed to be

$$
c_{k}=c_{k}^{(2)}=\sum_{j=0}^{k}\left(\begin{array}{l}
k \\
j
\end{array}\right)^{3} .
$$

This was then proved independently by Strehl (University of Erlangen-Nürnberg, see [13]), and myself. Strehl obtained the more general formula

$$
\sum_{k=0}^{n}\left(\begin{array}{l}
n \\
k
\end{array}\right)^{2} \frac{\left(\begin{array}{c}
\alpha+\beta+n+k \\
k
\end{array}\right)^{2}}{\left(\begin{array}{c}
\alpha+k \\
k
\end{array}\right)\left(\begin{array}{c}
\beta+k \\
k
\end{array}\right)}=\sum_{k=0}^{n}\left(\begin{array}{l}
n \\
k
\end{array}\right) \frac{\left(\begin{array}{c}
\alpha+\beta+n+k \\
k
\end{array}\right)}{\left(\begin{array}{c}
\beta+k \\
k
\end{array}\right)} \sum_{j=0}^{k}\left(\begin{array}{l}
k \\
j
\end{array}\right)^{2} \frac{\left(\begin{array}{c}
\beta+k \\
j
\end{array}\right)}{\left(\begin{array}{c}
\alpha+j \\
j
\end{array}\right)},
$$

where $\alpha$ and $\beta$ are parameters. The choice $\alpha=\beta=0$ gives the formula (1) for $\left(c_{k}\right)$ for $r=2$. In [13] Strehl also proved that $c_{k}^{(3)}$ is integral by establishing the formula

$$
c_{k}^{(3)}=\sum_{j=0}^{k}\left(\begin{array}{c}
k \\
j
\end{array}\right)^{2}\left(\begin{array}{c}
2 j \\
j
\end{array}\right)^{2}\left(\begin{array}{c}
2 j \\
k-j
\end{array}\right) .
$$

It is well known (see $[1,6])$ that Apéry's sequence

$$
a_{n}=a_{n}^{(2)}=\sum_{k=0}^{n}\left(\begin{array}{l}
n \\
k
\end{array}\right)^{2}\left(\begin{array}{c}
n+k \\
k
\end{array}\right)^{2}
$$

satisfies the recurrence relation

(3) $(n+1)^{3} a_{n+1}-\left((n+1)^{3}+n^{3}+4(2 n+1)^{3}\right) a_{n}+n^{3} a_{n-1}=0 \quad$ for $n \geq 0$.

The sequence (1) has also long been known to satisfy the recurrence relation (see $[1,2,3,5,6,12])$

$$
(k+1)^{2} c_{k+1}-\left(7 k^{2}+7 k+2\right) c_{k}-8 k^{2} c_{k-1}=0 \quad \text { for } k \geq 0 .
$$

After presenting some simple properties of the Legendre transform in Section 2, we consider in Section 3 a class of three term recurrent sequences $\left(c_{k}\right)$ such that the 
corresponding sequence $\left(a_{n}\right)$ is also three term recurrent. Simple examples of this kind are described in Section 4.

In Section 5 we consider the important recurrence (4) leading through Legendre transforms to Apéry's sequences related to $\zeta(3)$. In addition to obtaining the formula

$$
a_{n}^{(2)}=\sum_{k=0}^{n}\left(\begin{array}{l}
n \\
k
\end{array}\right)^{2}\left(\begin{array}{c}
n+k \\
k
\end{array}\right)^{2}=\sum_{k=0}^{n}\left(\begin{array}{l}
n \\
k
\end{array}\right)\left(\begin{array}{c}
n+k \\
k
\end{array}\right) \sum_{j=0}^{k}\left(\begin{array}{l}
k \\
j
\end{array}\right)^{3}
$$

we also get simultaneous approximations of $\pi^{2} / 8$ and $\zeta(3)$.

In Section 6 we consider the simpler sequence

$$
a_{n}=\sum_{k=0}^{n}\left(\begin{array}{c}
2 k \\
k
\end{array}\right)\left(\begin{array}{l}
n \\
k
\end{array}\right)\left(\begin{array}{c}
n+k \\
k
\end{array}\right)
$$

which is the Legendre transform of the sequence

$$
c_{k}=\sum_{j=0}^{k}\left(\begin{array}{l}
k \\
j
\end{array}\right)^{2}=\left(\begin{array}{c}
2 k \\
k
\end{array}\right) \text {. }
$$

This sequence is rather peculiar, namely

$$
\begin{aligned}
& a_{0}=1^{2}, \quad a_{1}=5 \cdot 1^{2}, \quad a_{2}=7^{2}, \quad a_{3}=5 \cdot 11^{2}, \quad a_{4}=91^{2}, \\
& a_{5}=5 \cdot 155^{2}, \quad a_{6}=1345^{2}, \quad a_{7}=5 \cdot 2365^{2}, \quad a_{8}=20995^{2}, \quad a_{9}=5 \cdot 37555^{2} \text {, }
\end{aligned}
$$

This will be explained by means of some particular sequences of orthogonal polynomials.

The final section contains a number of computer-aided results of recurrent sequences $\left(c_{k}\right)$ such that the corresponding sequence $\left(a_{n}\right)$ is also recurrent. We propose to continue this investigation by extending the class of recurrent sequences $\left(c_{k}\right)$ for which the corresponding sequence $\left(a_{n}\right)$ of Legendre transforms is known to be recurrent (see also [9]). Such insight might also prove the conjecture about $a_{n}^{(r)}$ for values of $r \geq 3$.

\section{Simple properties of Legendre transforms}

We shall mention the following simple results:

(i) If $\left(a_{n}\right)$ is the Legendre transform of $\left(c_{k}\right)$ then the following inversion formula holds:

$$
c_{k}=\sum_{j=0}^{k}(-1)^{k-j} \frac{2 j+1}{k+j+1} \frac{\left(\begin{array}{c}
k \\
j
\end{array}\right)}{\left(\begin{array}{c}
k+j \\
j
\end{array}\right)} a_{j} .
$$


(ii)

$$
\sum_{k=0}^{n} \frac{(-1)^{k}}{k+1}\left(\begin{array}{l}
n \\
k
\end{array}\right)\left(\begin{array}{c}
n+k \\
k
\end{array}\right)= \begin{cases}1 & n=0 \\
0 & n>0\end{cases}
$$

(iii) For $m \in \mathbb{N}$ we have

$$
\begin{aligned}
& \sum_{k=0}^{n} \frac{(-1)^{k}}{2 k-2 m+1}\left(\begin{array}{l}
n \\
k
\end{array}\right)\left(\begin{array}{c}
n+k \\
k
\end{array}\right)= \\
& (-1)^{m} \frac{(2 n+1-2(m-1)) \cdots(2 n-1)(2 n+1)(2 n+3) \cdots(2 n+1+2(m-1))}{(1 \cdot 3 \cdots(2 m-1))^{2}} .
\end{aligned}
$$

(iv) For $m \in \mathbb{N}$ we let

$$
c_{k}^{(m)}= \begin{cases}1 & k=m, \\ 0 & k \neq m .\end{cases}
$$

Then obviously

$$
\sum_{k=0}^{n} c_{k}^{(m)}\left(\begin{array}{l}
n \\
k
\end{array}\right)\left(\begin{array}{c}
n+k \\
k
\end{array}\right)=\frac{(n-m+1) \cdots(n-1) n(n+1) \cdots(n+m)}{(m !)^{2}} .
$$

Since

$$
\left(\begin{array}{l}
n \\
k
\end{array}\right)\left(\begin{array}{c}
n+k \\
k
\end{array}\right)=\left(\begin{array}{c}
2 k \\
k
\end{array}\right)\left(\begin{array}{l}
n+k \\
n-k
\end{array}\right)
$$

the relation (i) is an immediate consequence of the well-known relations for so-called Legendre pairs (cf. [8]):

$$
a_{n}=\sum_{k}\left(\begin{array}{c}
n+k \\
n-k
\end{array}\right) b_{k}
$$

if and only if

$$
b_{n}=\sum_{k}(-1)^{n+k}\left(\left(\begin{array}{c}
2 n \\
n-k
\end{array}\right)-\left(\begin{array}{c}
2 n \\
n-k-1
\end{array}\right)\right) a_{k}
$$

Notice that (ii) follows from (i), when applied to the sequence $\left(a_{n}\right)$ with $a_{0}=1, a_{n}=0$ for $n>0$. For another derivation of (ii) see [9]. Notice also that the formulas in (iii) and (iv) together give the inverse Legendre transform of an arbitrary polynomial sequence $\left(a_{n}\right)$.

\section{Three term recurrences and Legendre transforms}

We shall prove the following result: 
THEOREM 1. Let $A, B, C, D, E \in \mathbb{R}, C \neq 0$ be constants. We consider polynomials

$$
\begin{aligned}
& P_{0}(k)=A k^{2}+B k+C, \\
& P_{2}(k)=D k^{2}, \\
& Q_{1}(k)=k(A k+(B-A)), \\
& P_{1}(k)=D k(k+1)-Q_{1}(k)+E,
\end{aligned}
$$

and polynomials

$$
\begin{aligned}
& p_{0}(n)=(n+1) P_{0}(n), \\
& p_{2}(n)=n\left(P_{0}(n)-(B-A)(2 n+1)\right), \\
& q_{1}(n)=2 P_{1}(n)+2 Q_{1}(n)=2 D n(n+1)+2 E, \\
& p_{1}(n)=p_{0}(n)+p_{2}(n)+(2 n+1) q_{1}(n) .
\end{aligned}
$$

(i) Suppose the sequence $\left(c_{k}\right)$ satisfies the recurrence

$$
P_{0}(k) c_{k+1}-P_{1}(k) c_{k}-P_{2}(k) c_{k-1}=0 \quad \text { for } k \geq 1
$$

with initial values $c_{0}=1, c_{1}=E / C$. Then the Legendre transform $\left(a_{n}\right)$ of $\left(c_{k}\right)$ satisfies the recurrence

$$
p_{0}(n) a_{n+1}-p_{1}(n) a_{n}+p_{2}(n) a_{n-1}=0 \quad \text { for } n \geq 1
$$

with initial values $a_{0}=1, a_{1}=1+2 E / C$.

(ii) Suppose the sequence $\left(c_{k}\right)$ satisfies the recurrence (7) with initial values $c_{0}=0$, $c_{1}=1$. Then the Legendre transform $\left(a_{n}\right)$ of $\left(c_{k}\right)$ satisfies the recurrence

$$
p_{0}(n) a_{n+1}-p_{1}(n) a_{n}+p_{2}(n) a_{n-1}=C(4 n+2) \quad \text { for } n \geq 1
$$

with initial values $a_{0}=0, a_{1}=2$.

(iii) Suppose the sequence $\left(c_{k}\right)$ satisfies the recurrence

$$
P_{0}(k) c_{k+1}-P_{1}(k) c_{k}-P_{2}(k) c_{k-1}=\frac{(-1)^{k}}{k+1} \quad \text { for } k \geq 1
$$

with initial values $c_{0}=0, c_{1}=1 / C$. Then the Legendre transform $\left(a_{n}\right)$ of $\left(c_{k}\right)$ satisfies the recurrence (8) with initial values $a_{0}=0, a_{1}=2 / C$.

PROOF. For abbreviation we let

$$
a_{n, k}=c_{k}\left(\begin{array}{l}
n \\
k
\end{array}\right)\left(\begin{array}{c}
n+k \\
k
\end{array}\right)
$$

where $\left(c_{k}\right)$ is any sequence. The Legendre transform $\left(a_{n}\right)$ of $\left(c_{k}\right)$ is then given by

$$
a_{n}=\sum_{k=0}^{n} a_{n, k}
$$


We notice first that

(11) $p_{0}(n) a_{n+1, k}-p_{1}(n) a_{n, k}+p_{2}(n) a_{n-1, k}=$

$$
\begin{array}{r}
\left(2 p_{0}(n)\left(\begin{array}{c}
n \\
k-1
\end{array}\right)\left(\begin{array}{c}
n+k \\
k
\end{array}\right)-2 p_{2}(n)\left(\begin{array}{l}
n \\
k
\end{array}\right)\left(\begin{array}{c}
n+k-1 \\
k-1
\end{array}\right)\right. \\
\left.-(2 n+1) q_{1}(n)\left(\begin{array}{l}
n \\
k
\end{array}\right)\left(\begin{array}{c}
n+k \\
k
\end{array}\right)\right) c_{k} .
\end{array}
$$

Using the method of creative telescoping (cf. $[5,6])$ we let

$$
A_{n, k}=-\left(\begin{array}{c}
n \\
k
\end{array}\right)\left(\begin{array}{c}
n+k \\
k
\end{array}\right)(2 n+1)\left(\left(q_{1}(n)-2 Q_{1}(k)\right) c_{k}+2 P_{2}(k) c_{k-1}\right)
$$

for $0 \leq k \leq n$, and with the proviso that $A_{n, k}=0$ for $k<0$ or $k>n$. An easy rearrangement shows that identically for $0<k<n+1$ :

$$
\begin{array}{r}
A_{n, k}-A_{n, k-1}=\left(2 p_{0}(n)\left(\begin{array}{c}
n \\
k-1
\end{array}\right)\left(\begin{array}{c}
n+k \\
k
\end{array}\right)-2 p_{2}(n)\left(\begin{array}{l}
n \\
k
\end{array}\right)\left(\begin{array}{c}
n+k-1 \\
k-1
\end{array}\right)\right. \\
\left.-(2 n+1) q_{1}(n)\left(\begin{array}{l}
n \\
k
\end{array}\right)\left(\begin{array}{c}
n+k \\
k
\end{array}\right)\right) c_{k} \\
-2\left(\begin{array}{c}
n \\
k-1
\end{array}\right)\left(\begin{array}{c}
n+k-1 \\
k-1
\end{array}\right)(2 n+1) \times \\
\left(P_{0}(k-1) c_{k}-P_{1}(k-1) c_{k-1}-P_{2}(k-1) c_{k-2}\right) .
\end{array}
$$

In particular for $k=1$, and using (11), we also obtain

$$
\begin{aligned}
& A_{n, 1}-A_{n, 0}= \\
& \quad p_{0}(n) a_{n+1,1}-p_{1}(n) a_{n, 1}+p_{2}(n) a_{n \sim 1,1}-(4 n+2)\left(C c_{1}-E c_{0}\right) .
\end{aligned}
$$

We also notice that

$$
A_{n, 0}=-(2 n+1) q_{1}(n) c_{0} .
$$

Case 1. Assume first that $\left(c_{k}\right)$ satisfies (7) for $k \geq 1$. Then by (11) and (12)

$$
A_{n, k}-A_{n, k-1}=p_{0}(n) a_{n+1, k}-p_{1}(n) a_{n, k}+p_{2}(n) a_{n-1, k}
$$

for $1<k<n+1$. By (14) the relation (15.1) also holds for $k=0$. Using (7) we get

$$
\begin{aligned}
(16.1)-A_{n, n} & =\left(\begin{array}{c}
2 n \\
n
\end{array}\right)(2 n+1)\left(2 P_{1}(n) c_{n}+2 P_{2}(n) c_{n-1}\right)=2\left(\begin{array}{c}
2 n \\
n
\end{array}\right)(2 n+1) P_{0}(n) c_{n+1} \\
& =\left(\begin{array}{c}
2 n+2 \\
n+1
\end{array}\right)(n+1) P_{0}(n) c_{n+1}=p_{0}(n) a_{n+1, n+1},
\end{aligned}
$$


so that relation (15.1) also holds for $k=n+1$. Consequently by (13) and (15.1)

$$
\begin{aligned}
& p_{0}(n) a_{n+1}-p_{1}(n) a_{n}+p_{0}(n) a_{n-1} \\
& =\sum_{k=0}^{n+1}\left(A_{n, k}-A_{n, k-1}\right)+(4 n+2)\left(C c_{1}-E c_{0}\right) \\
& =(4 n+2)\left(C c_{1}-E c_{0}\right),
\end{aligned}
$$

which proves the two first claims of the theorem.

Case 2. Assume next that $\left(c_{k}\right)$ satisfies $(10)$ for $k \geq 1$ with $c_{0}=0, c_{1}=1 / C$. Then (15.1) is replaced by

$$
\begin{aligned}
A_{n, k}-A_{n, k-1}= & p_{0}(n) a_{n+1, k}-p_{1}(n) a_{n, k}+p_{2}(n) a_{n-1, k} \\
& -(4 n+2)\left(\begin{array}{c}
n \\
k-1
\end{array}\right)\left(\begin{array}{c}
n+k-1 \\
k-1
\end{array}\right) \frac{(-1)^{k-1}}{k}
\end{aligned}
$$

for $1<k<n+1$, and (16.1) is replaced by

$$
-A_{n, n}=p_{0}(n) a_{n+1, n+1}-(4 n+2)\left(\begin{array}{c}
2 n \\
n
\end{array}\right) \frac{(-1)^{n}}{n+1} .
$$

By (14) and (16.2) it follows that the relation (15.2) also holds for $k=n+1$ and also for $k=0$ when omitting the last term in (15.2). Since $C c_{1}-E c_{0}=1$ it follows by (13), (15.2) and Section 2(ii) that

$$
\begin{aligned}
& p_{0}(n) a_{n+1}-p_{1}(n) a_{n}+p_{2}(n) a_{n-1} \\
& =\sum_{k=0}^{n+1}\left(A_{n, k}-A_{n, k-1}\right)+(4 n+2) \sum_{k=0}^{n}\left(\begin{array}{l}
n \\
k
\end{array}\right)\left(\begin{array}{c}
n+k \\
k
\end{array}\right) \frac{(-1)^{k}}{k+1} \\
& =0 \quad \text { for } n>0 .
\end{aligned}
$$

This proves the last claim of the theorem.

REMARK 1. Assume that $B=2 A$, which implies that $p_{2}(n+1)=p_{0}(n)$. Assume further that $p_{0}(n) \neq 0$ for $n \geq 0$, and that $a_{n} \neq 0$ for $n \geq 0$. To distinguish the three sequences $\left(a_{n}\right)$ in $(i)-(i i i)$ they will here be denoted $\left(a_{n}\right),\left(a_{n}^{\prime}\right),\left(a_{n}^{\prime \prime}\right)$, respectively. The following formulas are easily deduced:

$$
\begin{gathered}
d_{n}^{\prime \prime}:=\left|\begin{array}{cc}
a_{n} & a_{n}^{\prime \prime} \\
a_{n+1} & a_{n+1}^{\prime \prime}
\end{array}\right|=\frac{2 C}{p_{0}(n)}, \\
D_{n}:=\left|\begin{array}{ccc}
a_{n-1} & a_{n-1}^{\prime} & a_{n-1}^{\prime \prime} \\
a_{n} & a_{n}^{\prime} & a_{n}^{\prime \prime} \\
a_{n+1} & a_{n+1}^{\prime} & a_{n+1}^{\prime \prime}
\end{array}\right|=\frac{-4 C^{2}(2 n+1)}{p_{0}(n-1) p_{0}(n)},
\end{gathered}
$$




$$
\begin{gathered}
d_{n}^{\prime}:=\left|\begin{array}{cc}
a_{n} & a_{n}^{\prime} \\
a_{n+1} & a_{n+1}^{\prime}
\end{array}\right|=\frac{2 C}{p_{0}(n)} \sum_{\nu=0}^{n}(2 v+1) a_{v}, \\
\alpha^{\prime \prime}:=\lim \frac{a_{n}^{\prime \prime}}{a_{n}}=2 C \sum_{n=0}^{\infty} \frac{1}{p_{0}(n) a_{n} a_{n+1}}, \\
\alpha^{\prime}:=\lim \frac{a_{n}^{\prime}}{a_{n}}=2 C \sum_{n=0}^{\infty} \frac{1}{p_{0}(n) a_{n} a_{n+1}} \sum_{\nu=0}^{n}(2 v+1) a_{v}, \\
\alpha^{\prime \prime}-\frac{a_{n}^{\prime \prime}}{a_{n}}=2 C \sum_{\nu=n}^{\infty} \frac{1}{p_{0}(\nu) a_{\nu} a_{v+1}}, \\
\alpha^{\prime}=\sum_{n=0}^{\infty}(2 n+1)\left(a_{n} \alpha^{\prime \prime}-a_{n}^{\prime \prime}\right) .
\end{gathered}
$$

The formulas concerning infinite series are purely formal, and convergence must therefore be ascertained when applied.

\section{Examples}

EXAMPLE 1. (Classical and generalized Legendre polynomials.) For $A=B=$ $D=0, \quad C=1, \quad E=x$ the recurrence

$$
c_{k+1}-x c_{k}-0 \cdot c_{k-1}=0
$$

has the solution $c_{k}=x^{k}$. The corresponding sequence

$$
a_{n}=\sum_{k=0}^{n}\left(\begin{array}{l}
n \\
k
\end{array}\right)\left(\begin{array}{c}
n+k \\
k
\end{array}\right) x^{k}
$$

then satisfies the recurrence

$$
(n+1) a_{n+1}-(2 n+1)(1+2 x) a_{n}+n a_{n-1}=0 .
$$

The polynomials $a_{n}=a_{n}(x)$ (Legendre polynomials) are orthogonal with respect to Lebesgue measure on $[-1,0]$.

For $A=B=0, C=1, D=x_{1}, E=x_{0}$ we get the recurrence

$$
c_{k+1}-\left(x_{0}+k(k+1) x_{1}\right) c_{k}-k^{2} x_{1} c_{k-1}=0 .
$$

The corresponding sequence of generalized Legendre polynomials $a_{n}$ then satisfies the recurrence

$$
(n+1) a_{n+1}-(2 n+1)\left(1+2\left(x_{0}+n(n+1) x_{1}\right) a_{n}+n a_{n-1}=0 .\right.
$$


Compare [9] for a wider class of generalized Legendre polynomials.

EXAMPLE 2. (Orthogonal polynomials related to Bernoulli numbers.) For $A=$ $D=0, B=C=1, E=x$ the recurrence

$$
(k+1) c_{k+1}-(x-k) c_{k}-0 \cdot c_{k-1}=0
$$

has the solution $c_{k}=\left(\begin{array}{l}x \\ k\end{array}\right)$. The corresponding sequence

$$
a_{n}=\sum_{k=0}^{n}\left(\begin{array}{l}
n \\
k
\end{array}\right)\left(\begin{array}{c}
n+k \\
k
\end{array}\right)\left(\begin{array}{l}
x \\
k
\end{array}\right)
$$

therefore satisfies the recurrence

$$
(n+1)^{2} a_{n+1}-(2 n+1)(1+2 x) a_{n}-n^{2} a_{n-1}=0 .
$$

When defining a linear functional $s$ on $\mathbb{R}[x]$ by

$$
s\left(\left(\begin{array}{l}
x \\
k
\end{array}\right)\right)=\frac{(-1)^{k}}{k+1}
$$

it follows easily by Section 2 (ii) and the recurrence relation that the polynomials $a_{n}=a_{n}(x)$ are orthogonal with respect to the functional $s$, and that

$$
s\left(a_{n}(x)^{2}\right)=\frac{(-1)^{n}}{2 n+1} .
$$

Since (compare [7])

$$
x^{n}=\sum_{k=0}^{n} A_{n k}\left(\begin{array}{l}
x \\
k
\end{array}\right)
$$

where

$$
A_{n k}=\sum_{j=0}^{k}(-1)^{j}\left(\begin{array}{l}
k \\
j
\end{array}\right)(k-j)^{n}=\sum_{j=0}^{k}(-1)^{k-j}\left(\begin{array}{l}
k \\
j
\end{array}\right) j^{n},
$$

it follows that

$$
s_{n}=s\left(x^{n}\right)=\sum_{k=0}^{n} A_{n k} \frac{(-1)^{k}}{k+1}=\sum_{k=0}^{n} \frac{1}{k+1} \sum_{j=0}^{k}(-1)^{j}\left(\begin{array}{c}
k \\
j
\end{array}\right) j^{n}=B_{n} .
$$

EXAMPLE 3. (Recurrent sequences related to Pell's equation.) Let $p$ be a prime number and $m \in \mathbb{N}$. Suppose that $\left(x_{1}, x_{2}\right) \in \mathbb{Z}^{2}$ is an arbitrary solution to Pell's equation

$$
x_{1}^{2}-p x_{2}^{2}=\varepsilon, \quad \varepsilon= \pm 1 .
$$


Let $A=1, B=0, C=-p^{2 m-1}$ be fixed. We consider two cases of values for $D$ and $E$ corresponding to a specific solution to Pell's equation:

Case (i). For $D=\varepsilon p x_{2}^{2}, E=-p^{m} x_{1} x_{2}-\varepsilon p^{2 m} x_{2}^{2}$, the recurrence (7) has the integral solution $c_{k}=D^{k}\left(x_{1} k+\varepsilon p^{m} x_{2}\right), k \geq 0$.

Case (ii). For $D=-\varepsilon x_{1}^{2}, E=-p^{m} x_{1} x_{2}+\varepsilon p^{2 m-1} x_{1}^{2}$, the recurrence (7) has the integral solution $c_{k}=D^{k}\left(x_{2} k-\varepsilon p^{m-1} x_{1}\right), k \geq 0$.

In particular for $C=-2$ (that is $p=2, m=1$ ) the fundamental solution $\left(x_{1}, x_{2}\right)=(1,1)$ with $\varepsilon=-1$ gives in case (i) $(D, E)=(-2,2)$, and thus the recurrence $\left(k^{2}-2\right) c_{k+1}+\left(3 k^{2}+k-2\right) c_{k}+2 k^{2} c_{k-1}=0$ has integral solution $c_{k}=$ $(-2)^{k}(k-2)$. The corresponding sequence

$$
a_{n}=\sum_{k=0}^{n}(-2)^{k}(k-2)\left(\begin{array}{l}
n \\
k
\end{array}\right)\left(\begin{array}{c}
n+k \\
k
\end{array}\right)
$$

therefore satisfies the recurrence

$$
(n+1)\left(n^{2}-2\right) a_{n+1}+(2 n+1)\left(3 n^{2}+3 n-2\right) a_{n}+n\left(n^{2}+2 n-1\right) a_{n-1}=0 .
$$

In case (ii), $(D, E)=(1,-4)$, and thus the recurrence

$$
\left(k^{2}-2\right) c_{k+1}-(2 k-4) c_{k}-k^{2} c_{k-1}=0
$$

has integral solution $c_{k}=k+1$. The corresponding sequence

$$
a_{n}=\sum_{k=0}^{n}(k+1)\left(\begin{array}{l}
n \\
k
\end{array}\right)\left(\begin{array}{c}
n+k \\
k
\end{array}\right)
$$

therefore satisfies the recurrence

$$
(n+1)\left(n^{2}-2\right) a_{n+1}-(2 n+1)\left(3 n^{2}+3 n-10\right) a_{n}+n\left(n^{2}+2 n-1\right) a_{n-1}=0 .
$$

Analogously for $C=-2$ the solution $\left(x_{1}, x_{2}\right)=(3,2)$ with $\varepsilon=1$ gives in case (i) $(D, E)=(8,-28)$, and thus the recurrence

$$
\left(k^{2}-2\right) c_{k+1}-\left(7 k^{2}+9 k-28\right) c_{k}-8 k^{2} c_{k-1}=0
$$

has integral solution $c_{k}=8^{k}(3 k+4)$. The corresponding sequence

$$
a_{n}=\sum_{k=0}^{n} 8^{k}(3 k+4)\left(\begin{array}{l}
n \\
k
\end{array}\right)\left(\begin{array}{c}
n+k \\
k
\end{array}\right)
$$

therefore satisfies the recurrence

$$
(n+1)\left(n^{2}-2\right) a_{n+1}-(2 n+1)\left(17 n^{2}+17 n-58\right) a_{n}+n\left(n^{2}+2 n-1\right) a_{n-1}=0 .
$$


In case (ii), $(D, E)=(-9,6)$, and thus the recurrence

$$
\left(k^{2}-2\right) c_{k+1}+\left(10 k^{2}+8 k-6\right) c_{k}+9 k^{2} c_{k-1}=0
$$

has integral solution $c_{k}=(-9)^{k}(2 k-3)$. The corresponding sequence

$$
a_{n}=\sum_{k=0}^{n}(-9)^{k}(2 k-3)\left(\begin{array}{l}
n \\
k
\end{array}\right)\left(\begin{array}{c}
n+k \\
k
\end{array}\right)
$$

therefore satisfies the recurrence

$$
(n+1)\left(n^{2}-2\right) a_{n+1}+(2 n+1)\left(17 n^{2}+17 n-10\right) a_{n}+n\left(n^{2}+2 n-1\right) a_{n-1}=0 .
$$

EXAMPLE 4. For $A=D=2, B=3, C=-2, E=-\frac{3}{2} x$ the recurrence is

$$
(k+2)(2 k-1) c_{k+1}-(k-3 x / 2) c_{k}-2 k^{2} c_{k-1}=0 .
$$

The corresponding sequence $\left(a_{n}\right)$ then satisfies the recurrence

$$
\begin{array}{r}
(n+1)(n+2)(2 n-1) a_{n+1}-(2 n+1)\left(2\left(n^{2}+n-1\right)+(2 n-1)(2 n+3) x\right) a_{n} \\
+n(n-1)(2 n+3) a_{n-1}=0
\end{array}
$$

The polynomials $a_{n}=a_{n}(x)$ are orthogonal with respect to a measure $\mu$ concentrated in the single point $x=-2 / 3$.

\section{Apéry's sequences}

By applying Theorem 1 for $A=C=1, B=E=2, D=8$, that is for

$$
P_{0}=(k+1)^{2}, \quad P_{1}=7 k^{2}+7 k+2, \quad P_{2}=8 k^{2},
$$

we get

THEOREM 2. (i) The Legendre transform $\left(a_{n}\right)$ of the sequence $\left(c_{k}\right)$ in (1) (which has $c_{0}=1, c_{1}=2$ ) satisfies the recurrence (3) with initial values $a_{0}=1, a_{1}=5$.

(ii) The Legendre transform $\left(a_{n}\right)$ of the sequence $\left(c_{k}\right)$ satisfying the recurrence (4) and having initial values $c_{0}=0, c_{1}=3$ satisfies the following recurrence

$$
\begin{array}{r}
(n+1)^{3} a_{n+1}-\left((n+1)^{3}+n^{3}+4(2 n+1)^{3}\right) a_{n}+n^{3} a_{n-1}= \\
3(4 n+2) \quad \text { for } n \geq 0
\end{array}
$$

with initial values $a_{0}=0, a_{1}=6$. 
(iii) The Legendre transform $\left(a_{n}\right)$ of the sequence $\left(c_{k}\right)$ satisfying the recurrence

$$
(k+1)^{2} c_{k+1}-\left(7 k^{2}+7 k+2\right) c_{k}-8 k^{2} c_{k-1}=\frac{(-1)^{k} 3}{k+1} \quad \text { for } k \geq 1
$$

and having initial values $c_{0}=0, c_{1}=3$ satisfies the recurrence (3) for $n \geq 1$ with initial values $a_{0}=0, a_{1}=6$.

REMARK 2. . As in Remark 1 we denote the three sequences $\left(a_{n}\right)$ in (i)-(iii) by $\left(a_{n}\right),\left(a_{n}^{\prime}\right),\left(a_{n}^{\prime \prime}\right)$, respectively, and similarly for the sequences $\left(c_{k}\right)$.

Since $B=2 A$ the formulas in Remark 1 applies with $p_{0}(n)=(n+1)^{3}$. In this case it is well known (cf. [6]) that

$$
\lim \frac{c_{k}^{\prime}}{c_{k}}=\pi^{2} / 8, \quad \alpha^{\prime \prime}:=\lim \frac{a_{n}^{\prime \prime}}{a_{n}}=\zeta(3)
$$

Therefore also

$$
\alpha^{\prime}:=\lim \frac{a_{n}^{\prime}}{a_{n}}=\pi^{2} / 8, \quad \lim \frac{c_{k}^{\prime \prime}}{c_{k}}=\zeta(3) .
$$

The simultaneous approximation of $\pi^{2} / 8$ and $\zeta(3)$ is illustrated in the following two tables:

\begin{tabular}{|l|l|l|l|l|l|}
\hline$k$ & $c_{k}$ & $c_{k}^{\prime}$ & $c_{k}^{\prime \prime}$ & $\pi^{2} / 8-c_{k}^{\prime} / c_{k}$ & $\zeta(3)-c_{k}^{\prime \prime} / c_{k}$ \\
\hline 0 & 1 & 0 & 0 & 1.233700550 & 1.202056903 \\
1 & 2 & 3 & 3 & -0.266299450 & -0.297943097 \\
2 & 10 & 12 & $93 / 8$ & 0.033700550 & 0.039556903 \\
3 & 56 & $208 / 3$ & $1217 / 18$ & -0.004394688 & -0.005284367 \\
4 & 346 & $1280 / 3$ & $239429 / 576$ & 0.000559895 & 0.000683067 \\
\hline
\end{tabular}

\begin{tabular}{|l|l|l|l|c|c|}
\hline$n$ & $a_{n}$ & $a_{n}^{\prime}$ & $a_{n}^{\prime \prime}$ & $\pi^{2} / 8-a_{n}^{\prime} / a_{n}$ & $\zeta(3)-a_{n}^{\prime \prime} / a_{n}$ \\
\hline 0 & 1 & 0 & 0 & 1.233700550 & 1.202056903 \\
1 & 5 & 6 & 6 & 0.033700550 & 0.002056903 \\
2 & 73 & 90 & $351 / 4$ & 0.000823838 & 0.000002109 \\
3 & 1445 & $5348 / 3$ & $62531 / 36$ & 0.000021196 & 0.000000002 \\
4 & 33001 & $122140 / 3$ & $11424695 / 288$ & 0.000000561 & 0.000000000 \\
\hline
\end{tabular}




\section{A peculiar sequence}

We consider now the sequence $a_{n}=\sum_{k=0}^{n}\left(\begin{array}{c}2 k \\ k\end{array}\right)\left(\begin{array}{c}n \\ k\end{array}\right)\left(\begin{array}{c}n+k \\ k\end{array}\right)$. To explain the properties mentioned in the introduction we consider the following sequences of polynomials

$$
\begin{aligned}
& a_{n}(x)=\sum_{k=0}^{n}\left(\begin{array}{c}
2 k \\
k
\end{array}\right)\left(\begin{array}{l}
n \\
k
\end{array}\right)\left(\begin{array}{c}
n+k \\
k
\end{array}\right) x^{k}, \\
& P_{n}^{-}(x)=\sum_{j=0}^{n}\left(\begin{array}{l}
n \\
j
\end{array}\right)\left(\begin{array}{c}
n+j-\frac{1}{2} \\
j
\end{array}\right) x^{j}, \\
& P_{n}^{+}(x)=\sum_{j=0}^{n}\left(\begin{array}{l}
n \\
j
\end{array}\right)\left(\begin{array}{c}
n+j+\frac{1}{2} \\
j
\end{array}\right) x^{j} .
\end{aligned}
$$

Then we claim that

$$
\begin{aligned}
a_{2 n}(x) & =P_{n}^{-}(4 x)^{2}, \\
a_{2 n+1}(x) & =(1+4 x) P_{n}^{+}(4 x)^{2} .
\end{aligned}
$$

Since $a_{n}=a_{n}(1)$ we get in particular

$$
a_{2 n}=P_{n}^{-}(4)^{2}, \quad a_{2 n+1}=5 P_{n}^{+}(4)^{2},
$$

which explains the peculiarities of the sequence $a_{n}$.

The polynomials $P_{n}^{-}(x)$ and $P_{n}^{+}(x)$ are expressible in terms of the Jacobi polynomials

$$
P_{n}^{(\alpha, \beta)}(x)=\frac{(\alpha+1)_{n}}{n !} F[-n, n+\alpha+\beta+1 ; \alpha+1 ;(1-x) / 2]
$$

In fact

$$
\begin{aligned}
& P_{n}^{-}(x)=P_{n}^{\left(0,-\frac{1}{2}\right)}(2 x+1)=F\left[-n, n+\frac{1}{2} ; 1 ;-x\right], \\
& P_{n}^{+}(x)=P_{n}^{\left(0,+\frac{1}{2}\right)}(2 x+1)=F\left[-n, n+\frac{3}{2} ; 1 ;-x\right] .
\end{aligned}
$$

Therefore formula (22) follows immediately from Clausen's formula (cf. [11])

$$
F^{2}\left[a, b ; a+b+\frac{1}{2} ; z\right]={ }_{3} F_{2}\left[2 a, 2 b, a+b ; 2 a+2 b, a+b+\frac{1}{2} ; z\right]
$$

with $a=-n, b=n+\frac{1}{2}, z=-4 x$.

The polynomial sequence $u_{n}=P_{n}^{-}(x)$ satisfies the recurrence relation

$$
\begin{aligned}
(n+1)(2 n+1)(4 n-1) u_{n+1}-(4 n+1) & \left(\frac{1}{2}(4 n-1)(4 n+3) x+4 n^{2}+2 n-1\right) u_{n} \\
(25) & +n(2 n-1)(4 n+3) u_{n-1}=0 \text { for } n \geq 0,
\end{aligned}
$$


and is thus (cf. [14]) a sequence of orthogonal polynomials with respect to a normalized measure $\mathrm{m}^{-}$on $\mathbb{R}$. The measure is given by

$$
d m^{-}(t)= \begin{cases}d t / 2 \sqrt{1+t}, & t \in]-1,0] \\ 0 & \text { otherwise }\end{cases}
$$

the corresponding moments being

$$
\mu_{n}^{-}=\frac{(-1)^{n} 4^{n}}{(2 n+1)\left(\begin{array}{c}
2 n \\
n
\end{array}\right)}, \quad n \geq 0 .
$$

Also

$$
\left\|P_{n}^{-}(x)\right\|^{2}=\frac{1}{4 n+1}, \quad n \geq 0 .
$$

Analogously formula (22) follows from a more general formula of Orr (see [11, Theorem III]), and contiguous relations.

Similarly the polynomial sequence $u_{n}=P_{n}^{+}(x)$ satisfies the recurrence relation

$$
\begin{aligned}
& (n+1)(2 n+3)(4 n+1) u_{n+1}-(4 n+3)\left(\frac{1}{2}(4 n+1)(4 n+5) x+4 n^{2}+6 n+1\right) u_{n} \\
& (29) \quad+n(2 n+1)(4 n+5) u_{n-1}=0 \quad \text { for } n \geq 0,
\end{aligned}
$$

and is a sequence of orthogonal polynomials with respect to a normalized measure $m^{+}$on $\mathbb{R}$. The measure is given by

$$
d m^{+}(t)= \begin{cases}\frac{3}{2} \sqrt{1+t} d t, & t \in]-1,0] \\ 0 & \text { otherwise }\end{cases}
$$

the corresponding moments being

$$
\mu_{n}^{+}=\frac{(-1)^{n} 3 \cdot 4^{n}}{(2 n+1)(2 n+3)\left(\begin{array}{c}
2 n \\
n
\end{array}\right)}, \quad n \geq 0 .
$$

Also

$$
\left\|P_{n}^{+}(x)\right\|^{2}=\frac{3}{4 n+3}, \quad n \geq 0
$$

\section{Some computer-aided results}

We shall mention some further examples of recurrences for Legendre transforms.

EXAMPLE 5. For the sequence (cf. Section 6) $c_{k}=\left(\begin{array}{c}2 k \\ k\end{array}\right) x^{k}$ and satisfying the recurrence

$$
(k+1) c_{k+1}-(4 k+2) x c_{k}=0,
$$


the corresponding Legendre transform $\left(a_{n}\right)$ satisfies the four term recurrence

$$
\begin{aligned}
& (2 n+1)(n+2)^{2} a_{n+2}-(2 n+3)\left(3 n^{2}+6 n+2+4(2 n+1)(2 n+3) x\right) a_{n+1} \\
& \quad+(2 n+1)\left(3 n^{2}+6 n+2+4(2 n+1)(2 n+3) x\right) a_{n}-(2 n+3) n^{2} a_{n-1}=0 .
\end{aligned}
$$

This follows by a telescopic argument using

$$
A_{n, k}=-4(2 n+1)(2 n+3)\left(\begin{array}{c}
n+1 \\
k
\end{array}\right)\left(\begin{array}{c}
n+k \\
k
\end{array}\right)(4 k+2) x c_{k} .
$$

EXAMPLE 6. For the sequence $\left(c_{k}\right)$ of Fibonacci numbers with $c_{0}=c_{1}=1$ and satisfying the recurrence

$$
c_{k+1}-c_{k}-c_{k-1}=0,
$$

the corresponding Legendre transform $\left(a_{n}\right)$ satisfies the five term recurrence

$$
\begin{aligned}
& (2 n-1)(n+1)(n+2) a_{n+2}-4(2 n-1)(2 n+3)(n+1) a_{n+1} \\
& \quad-2(2 n+1)\left(n^{2}+n-1\right) a_{n}-4(2 n-1)(2 n+3) n a_{n-1} \\
& \quad+(2 n+3)(n-1) n a_{n-2}=0 .
\end{aligned}
$$

This follows by a telescopic argument using

$$
A_{n, k}=A_{n, 0}\left(\begin{array}{c}
n \\
k-1
\end{array}\right)\left(\begin{array}{c}
n+k-1 \\
k-1
\end{array}\right) \frac{1}{k^{2}}\left(\left(n^{2}+n-k(k-1)\right) c_{k}+k^{2} c_{k-1}\right)
$$

with

$$
A_{n, 0}=-4(2 n-1)(2 n+1)(2 n+3) .
$$

EXAMPLE 7. For the sequence $\left(c_{k}\right)$ of Legendre polynomials satisfying the recurrence

$$
(k+1) c_{k+1}-(2 k+1)(2 x+1) c_{k}+k c_{k-1}=0,
$$

the corresponding Legendre transform $\left(a_{n}\right)$ satisfies the five term recurrence

$$
\begin{aligned}
& (2 n-1)(n+2)^{2} a_{n+2}-(3+4 x)(2 n-1)(2 n+3)^{2} a_{n+1} \\
& \quad+(2 n+1)\left(38 n^{2}+38 n-29+8(2 n-1)(2 n+3) x\right) a_{n} \\
& \quad-(3+4 x)(2 n-1)^{2}(2 n+3) a_{n-1}+(2 n+3)(n-1)^{2} a_{n-2}=0 .
\end{aligned}
$$

This follows by a telescopic argument using

$A_{n, k}=A_{n, 0}\left(\begin{array}{c}n \\ k-1\end{array}\right)\left(\begin{array}{c}n+k-1 \\ k-1\end{array}\right) \frac{1}{k^{2}}\left(\left(n^{2}+n-k(k-1)-k(2 k+1)(2 x+1)\right) c_{k}+k^{2} c_{k-1}\right)$

with

$$
A_{n, 0}=4(2 n-1)(2 n+1)(2 n+3) .
$$


EXAMPLE 8. For a sequence $\left(c_{k}\right)$ satisfying a recurrence

$$
P_{0}(k) c_{k+1}-P_{1}(k) x c_{k}-P_{2}(k) x^{2} c_{k-1}=0,
$$

for $k \geq 0$, where

$$
P_{0}(k)=(k+1)^{2}, \quad P_{1}(k)=\alpha k^{2}+\alpha k+\beta, \quad P_{2}(k)=\gamma k^{2},
$$

the corresponding Legendre transform $\left(a_{n}\right)$ satisfies the five term recurrence

$$
\begin{aligned}
& (2 n-1) n(n+2)^{3} a_{n+2} \\
& \quad-(2 n-1)(2 n+3)\left(2 n^{3}+6 n^{2}+4 n-1+2 n P_{1}(n+1) x\right) a_{n+1} \\
& \quad-(2 n+1)\left(4 \gamma(2 n-1)(2 n+3) n(n+1) x^{2}\right. \\
& \left.\quad-2(2 n-1)(2 n+3)\left(P_{1}(n)-2 P_{1}(0)\right) x-\left(6 n^{4}+12 n^{3}-2 n^{2}-8 n+3\right)\right) a_{n} \\
& \quad-(2 n-1)(2 n+3)\left(2 n^{3}-2 n+1+2(n+1) P_{1}(n-1) x\right) a_{n-1} \\
& \quad+(2 n+3)(n+1)(n-1)^{3} a_{n-2}=0 .
\end{aligned}
$$

This follows by a telescopic argument using

$$
\begin{aligned}
A_{n, k}=- & 4(2 n-1)(2 n+1)(2 n+3)\left(\begin{array}{c}
n+1 \\
k
\end{array}\right)\left(\begin{array}{c}
n+k-1 \\
k
\end{array}\right) \\
& \times\left(\left(\gamma\left(n^{2}+n-k(k-1)\right) x^{2}+P_{1}(k) x\right) c_{k}+P_{2}(k) x^{2} c_{k-1}\right) .
\end{aligned}
$$

Important examples are

$$
c_{k}=\sum_{j=0}^{k}\left(\begin{array}{l}
k \\
j
\end{array}\right)^{3} x^{k}
$$

satisfying the above recurrence with $(\alpha, \beta, \gamma)=(7,2,8)$, and

$$
c_{k}=\sum_{j=0}^{k}\left(\begin{array}{l}
k \\
j
\end{array}\right)^{2}\left(\begin{array}{c}
k+j \\
j
\end{array}\right) x^{k}
$$

satisfying the above recurrence with $(\alpha, \beta, \gamma)=(11,3,1)$ (cf. [6]).

EXAMPLE 9. For the sequence

$$
c_{k}=\sum_{j=0}^{k}\left(\begin{array}{l}
k \\
j
\end{array}\right)^{4} x^{k},
$$

satisfying the three term recurrence (see $[1,2,4,5,6,12]$ )

$$
P_{0}(k) c_{k+1}-P_{1}(k) x c_{k}-P_{2}(k) x^{2} c_{k-1}=0,
$$


where

$P_{0}(k)=(k+1)^{3}, \quad P_{1}(k)=2(2 k+1)\left(3 k^{2}+3 k+1\right), \quad P_{2}(k)=(4 k-1) 4 k(4 k+1)$, the corresponding Legendre transform $\left(a_{n}\right)$ satisfies the seven term recurrence

$$
\begin{aligned}
& (2 n-3)(2 n-1) n(n+3)^{4} a_{n+3} \\
& -(2 n-1)(2 n-3)(2 n+5)\left(3 n^{4}+22 n^{3}+52 n^{2}+33 n-16\right. \\
& \left.+2 n P_{1}(n+2) x\right) a_{n+2} \\
& -(2 n-3)(2 n+3)\left(\left(1024 n^{5}+6144 n^{4}+10944 n^{3}+2944 n^{2}-5040 n\right) x^{2}\right. \\
& -\left(192 n^{5}+1104 n^{4}+1728 n^{3}-180 n^{2}-1332 n+420\right) x \\
& \left.-\left(15 n^{5}+85 n^{4}+120 n^{3}-60 n^{2}-126 n+80\right)\right) a_{n+1} \\
& +2(2 n-3)(2 n+1)(2 n+5)\left(8(2 n-1)(2 n+3)\left(16 n^{2}+16 n-15\right) x^{2}\right. \\
& \left.-\left(72 n^{4}+144 n^{3}-98 n^{2}-170 n+126\right) x-\left(5 n^{4}+10 n^{3}-10 n^{2}-15 n+16\right)\right) a_{n} \\
& -(2 n-1)(2 n+5)\left(\left(1024 n^{5}-1024 n^{4}-3392 n^{3}+3264 n^{2}+2448 n-2160\right) x^{2}\right. \\
& -\left(192 n^{5}-144 n^{4}-768 n^{3}+660 n^{2}+756 n-756\right) x \\
& \left.-\left(15 n^{5}-10 n^{4}-70 n^{3}+60 n^{2}+89 n-96\right)\right) a_{n-1} \\
& -(2 n-3)(2 n+3)(2 n+5)\left(3 n^{4}-10 n^{3}+4 n^{2}+17 n-16\right. \\
& \left.+2(n+1) P_{1}(n-2) x\right) a_{n-2} \\
& +(2 n+3)(2 n+5)(n+1)(n-2)^{4} a_{n-3}=0 .
\end{aligned}
$$

This follows by a telescopic argument using

$$
\begin{array}{r}
A_{n, k}=-8(2 n-3)(2 n-1)(2 n+1)(2 n+3)(2 n+5)\left(\begin{array}{c}
n+1 \\
k-1
\end{array}\right)\left(\begin{array}{c}
n+k-2 \\
k-1
\end{array}\right) \frac{1}{k^{2}} \\
\times\left(4(4 k+3)(4 k+5)\left(n^{2}+n-(k-1)(k-2)\right) x^{2} c_{k}\right. \\
\left.+k\left(P_{1}(k) x c_{k}+P_{2}(k) x^{2} c_{k-1}\right)\right)
\end{array}
$$

with

$$
A_{n, 0}=-480(2 n-3)(2 n-1)(2 n+1)(2 n+3)(2 n+5) .
$$

The computations were performed by means of the GP-PARI system using the methods in [5].

Added in proof. It has been pointed out to me by Michael Stoll (University of Bonn) that arguments taken from R. P. Stanley, 'Differentiably finite power series', European J. Combin. 7 (1980), 175-188, lead to the result (illustrated by the examples above) that the set of polynomially recursive sequences is invariant under the Legendre transform and the inverse Legendre transform. 


\section{References}

[1] R. Askey and J. A. Wilson, 'A recurrence relation generalizing those of Apéry', J. Austral. Math. Soc. (Series A) 36 (1984), 267-278.

[2] T. Cusick, 'Recurrences for sums of powers of binomial coefficients', J. Combin. Theory Ser. A 52 (1989), 77-83.

[3] J. Franel, L'Intermédiaire des Mathématiciens vol. I (1894).

[4] —_ L'Intermédiaire des Mathématiciens vol. 2 (1895).

[5] M. A. Perlstadt, 'Some recurrences for sums of powers of binomial coefficients', J. Number Theory 27 (1987), 304-309.

[6] A. J. van der Poorten, 'A proof that Euler missed ... A Apéry's proof of the irrationality of $\zeta(3)$ ', Math. Intelligencer 1 (1978/79), 195-203.

[7] H. Rademacher, Topics in analytic number theory (Springer, Berlin, 1973).

[8] J. Riordan, Combinatorial identities (Wiley, New York, 1968).

[9] A. L. Schmidt, 'Generalized Legendre polynomials', J. Reine Angew. Math. 404 (1990), 192-202.

[10] - 'Generalized q-Legendre polynomials', J. Comput. Appl. Math. 49 (1993), 243-249.

[11] L. J. Slater, Generalized hypergeometric functions (Cambridge Univ. Press, London, 1966).

[12] T. B. Staver, 'Om summasjon av potenser av binomialkoefficientene', Norsk Matematisk Tidskrift 29 (1947), 97-103.

[13] V. Strehl, 'Binomial identities - combinatorial and algorithmic aspects', Discrete Math. 136 (1994), 309-346.

[14] G. Szegö, Orthogonal polynomials (Amer. Math. Soc., Providence, 1975).

\section{Matematisk Institut \\ Universitetsparken 5 \\ DK-2100 Copenhagen $\emptyset$ \\ Denmark}

